\title{
The limitations of manually entered data in acute care environments
}

\author{
Jesse M. Ehrenfeld, MD · Jonathan P. Wanderer, MD
}

Received: 7 November 2014 / Accepted: 10 November 2014/Published online: 15 November 2014

(C) Canadian Anesthesiologists' Society 2014

\section{To the Editor,}

Avidan et al. have found variable accuracy in manually entered drug administration data, including prospective observation of drug omissions, drug entries in the perioperative information management systems (PIMS) where drug administration was not observed, and discrepancies in timing. ${ }^{1}$ These findings are concerning given that the cornerstone of PIMS functionality is the creation of an accurate and timely intraoperative record. ${ }^{2}$

In their discussion, Avidan et al. note that most of the drug omissions represented administration of small doses of hypnotic agents or vasoactive drugs. The authors hypothesize that "the occurrence of these omissions during maintenance may suggest that they were not related to the anesthesiologist's activity level but rather to the drug doses being perceived as non-significant or, alternatively, as self-incriminating.,"

These findings are consistent with data we published in 2011 arising from a comparison of paper $(n=500) v s$ electronic anesthesia records $(n=161,210)$ and an evaluation of gaps in the measurement of intraoperative vital signs. ${ }^{3}$ Although $5.4 \%$ of the electronic records we evaluated from three different hospitals documented at least one ten-minute gap in blood pressure measurement during an anesthetic case, a manual review of 500 paper anesthesia records in our study did not reveal a single case (99\% upper confidence limit for blood pressure gaps of $0.83 \%$ ). At the time, we hypothesized that the absence of gaps in vital signs in the paper anesthesia records reflected

J. M. Ehrenfeld, MD ( $\square)$ · J. P. Wanderer, MD

Departments of Anesthesiology, Surgery, Biomedical

Informatics, and Health Policy, Vanderbilt University, Nashville,

TN, USA

e-mail: jesse.ehrenfeld@vanderbilt.edu "interpolation" of missing values by the anesthesia provider.

"Smoothing" vital signs during the recording of manual anesthesia records is a well-described problem, ${ }^{4}$ and there is no question in our minds that electronically captured data are superior in quality compared with manually entered data. Nevertheless, these discrepancies raise the issue of how credible electronic records may be when they contain manually entered drug administration or vital signs data. ${ }^{5}$

This work by Avidan et al. is another reminder of the limitations of manually recorded data in acute care environments. There are emerging solutions to these shortcomings, including wireless pumps that transmit drug infusion rates directly into an electronic record and systems to record drug administration automatically. ${ }^{\mathrm{A}}$ While it is unlikely that human performance will ever match that of automated systems, we must ask ourselves this question: Is the current level of accuracy adequate? If the answer is no, we must commit ourselves to developing and implementing said solutions.

Conflicts of interest None declared.

\section{References}

1. Avidan A, Dotan K, Weissman C, Cohen MJ, Levin PD. Accuracy of manual entry of drug administration data into an anesthesia information management system. Can J Anesth 2014; 61: 979-85.

2. Ehrenfeld JM, Rehman MA. Anesthesia information management systems: a review of functionality and installation considerations. J Clin Monit Comput 2011; 25: 71-9.

\footnotetext{
A BD Manufacturer. Available from URL: www.bd.com/intelliport (accessed November 2014).
} 
3. Ehrenfeld JM, Epstein RH, Bader S, Kheterpal S, Sandberg WS. Automatic notifications mediated by anesthesia information management systems reduce the frequency of prolonged gaps in blood pressure documentation. Anesth Analg 2011; 113: 356-63.
4. Reich DL, Wood RK Jr, Mattar R, et al. Arterial blood pressure and heart rate discrepancies between handwritten and computerized anesthesia records. Anesth Analg 2000; 91: 612-6. 5. Reich DL, Wax DB. Mind the gap. Anesth Analg 2011; 113: 214-5. 